\title{
A new catalogue of ISM content of normal galaxies $\star$
}

\author{
D. Bettoni ${ }^{1}$, G. Galletta ${ }^{2}$, and S. García-Burillo ${ }^{3}$ \\ 1 Osservatorio Astronomico di Padova, Vicolo Osservatorio 5, 35122 Padova, Italy \\ 2 Dipartimento di Astronomia, Università di Padova, Vicolo Osservatorio 2, 35122 Padova, Italy \\ 3 Observatorio Astronómico Nacional-OAN, Apartado 1143, 28800 Alcalá de Henares, Madrid, Spain
}

Received 19 February 2003 / Accepted 1 April 2003

\begin{abstract}
We have compiled a catalogue of the gas content for a sample of 1916 galaxies, considered to be a fair representation of "normality". The definition of a "normal" galaxy adopted in this work implies that we have purposely excluded from the catalogue galaxies having distorted morphology (such as interaction bridges, tails or lopsidedness) and/or any signature of peculiar kinematics (such as polar rings, counterrotating disks or other decoupled components). In contrast, we have included systems hosting active galactic nuclei (AGN) in the catalogue. This catalogue revises previous compendia on the ISM content of galaxies published by Bregman et al. (1992) and Casoli et al. (1998), and compiles data available in the literature from several small samples of galaxies. Masses for warm dust, atomic and molecular gas, as well as X-ray luminosities have been converted to a uniform distance scale taken from the Catalogue of Principal Galaxies (PGC). We have used two different normalization factors to explore the variation of the gas content along the Hubble sequence: the blue luminosity $\left(L_{\mathrm{B}}\right)$ and the square of linear diameter $\left(D_{25}^{2}\right)$. Our catalogue significantly improves the statistics of previous reference catalogues and can be used in future studies to define a template ISM content for "normal" galaxies along the Hubble sequence. The catalogue can be accessed on-line and is also available at the Centre des Données Stellaires (CDS).
\end{abstract}

Key words. catalogs - galaxies: ISM - galaxies: general - infrared: galaxies - radio lines: galaxies - X-rays: galaxies submillimeter

\section{Introduction}

Different surveys have been conducted thus far to establish whether galaxies can be characterized by a canonical gas content that may vary significantly along the Hubble sequence (early-type versus late-type objects) and/or depend on the particular environment (isolated versus strongly interacting galaxies) (Knapp et al. 1989; Bregman et al. 1992; Casoli et al. 1998; Fabbiano et al. 1992; Burstein et al. 1997; Beuing et al. 1999). A global study of the ISM content in galaxies requires a multi-wavelength approach: far infrared (FIR) observations at 60 and $100 \mu \mathrm{m}$ trace the warm dust content, while those at $140-200 \mu \mathrm{m}$ the cold dust; mm lines of carbon monoxide (CO) and the $21 \mathrm{~cm}$ line of HI must be used to infer the molecular and atomic gas content, respectively: the X-ray wave-band is used to study the hot gas component. However, results obtained from previous surveys have strong biases due to poor statistics (uneven coverage along the Hubble sequence) and, also, due to the inclusion of galaxies which are known to be

\footnotetext{
Send offprint requests to: G. Galletta, e-mail: galletta@pd.astro.it

* The catalogue is available in electronic form at http://dipastro.pd.astro.it/galletta/ismcat and at the CDS via anonymous ftp to

cdsarc.u-strasbg.fr $(130.79 .128 .5)$ or via

http://cdsweb.u-strasbg. fr/cgi-bin/qcat? J/A+A/405/5
}

"peculiar". As an example, the selection of candidate galaxies to be observed in CO surveys have been typically based on Far Infrared Luminosity (FIR) criteria. This often favors the inclusion of "peculiar" galaxies in surveys and introduces a bias against "normal" systems.

In this work we present a new catalogue describing the Interstellar Medium (ISM) content in a sample of 1916 "normal" galaxies. A first preliminary version of this catalogue was used by Bettoni et al. (2001) (Paper I) as a reference in their study of the gas content of gas-accreting galaxies. First of all, this requires the adoption of a non-obvious definition for "normality". In this work we will consider a galaxy to be "peculiar", hence to be excluded from the catalogue, if it has any sign of perturbed morphology due to a recent or ongoing interaction like tidal tails, bridges, warps or disk lopsidedness. Furthermore, with our definition of normal galaxy, we also exclude from this catalogue any galaxy having any sign of peculiar kinematics, revealed by the presence of polar rings, counterrotating cores/disks or other decoupled components. With this definition, we do not exclude in principle galaxies belonging to groups or clusters: here, a "normal" galaxy is not necessarily an "isolated" galaxy, but rather a dynamically relaxed stable system. Nuclear activity is far from universal among galaxies, although most galaxies are known to host super-massive black holes in their nuclei. We purposely include active galaxies in our sample, considering that activity 
may represent just an episodic stage in the evolution of normal galaxies that appear as globally unperturbed, and therefore fitting our requirements.

The ISM catalogue has been compiled making use of several sources available in the literature. All values have been re-scaled using a common distance reference taken from the Catalogue of Principal Galaxies (PGC). We derive mean values for the ISM content along the Hubble sequence, using a survival analysis method that properly takes into account upper limits. The various gas content estimators have been normalized using both the galaxy absolute blue luminosity $L_{\mathrm{B}}$ and the size $D_{25}^{2}$. The latter allows the direct comparison of our results with those of previous works that used either $L_{\mathrm{B}}$ (Bregman et al. 1992) or $D_{25}^{2}$ (Casoli et al. 1998) to derive canonical mean values for galaxies of different Hubble types.

The new catalogue significantly improves the statistics of previous reference catalogues. This is especially relevant in the case of early type galaxies: compared to previous studies, our sample improves the number of early-type galaxies included by a factor $>3$ (from ellipticals to lenticulars). This catalogue is intended to be used in future studies as a reference for the ISM content in normal galaxies. The catalogue can be accessed on line and is also available at the Centre des Données Stellaires (CDS).

\section{Data compilation}

\subsection{The data search}

We searched in the literature for the different available papers (both survey papers and those dealing with case-by-case studies (Knapp et al. 1989; Roberts et al. 1991; Casoli et al. 1998; Fabbiano et al. 1992; Burstein et al. 1997; Beuing et al. 1999)) and collected all the published data on the different tracers of ISM in galaxies. This produced a preliminary list of more than 3400 candidate galaxies. From this list, all the systems having an acknowledged peculiarity had to be excluded. In addition, we found 148 galaxies, included in this preliminary compilation, that are listed in the Veron et al. (2000) catalogue of active galaxy nuclei (AGN). For the reasons explained above, we decided to include these galaxies in our sample, although they will be discussed separately.

\subsection{Discarded types of galaxies}

We considered all the systems belonging to at least one of the following categories to be globally peculiar and we therefore removed them from our sample:

1) Interacting and disturbed galaxies. They are mainly listed in the Arp (1966), Arp \& Madore (1987) and Vorontsov-Velyaminov (1959) catalogues and appear to be interacting with a close object or have an evident sign of distortion in their disks/spheroids. This category includes 1422 galaxies, i.e., the bulk of the systems we discarded to build this compilation.

2) Polar ring galaxies. Some galaxies appear to host polaror very inclined-rings of gas and/or stars. In this category of peculiar galaxies we find ellipticals (Bertola \& Galletta 1978; Hawarden et al. 1981) such as NGC 5128 and disk galaxies (Schweizer et al. 1983; Whitmore et al. 1990) as NGC 4650A. These decoupled components betray a passed accretion episode. The origin of polar rings is connected to an interaction or merging between a galaxy and a "donor" source which may be another galaxy or intergalactic gas. The ISM content of polar rings has been discussed in Paper I. We found 51 polar ring galaxies, which were removed from our catalogue.

3) Galaxies with counterrotation. Counterrotating galaxies have a significant amount of their gas and/or stars (Galletta 1996; Bettoni et al. 2001) rotating in opposite direction with respect to the majority of the stars. Similarly to the case of polar rings, the counterrotation phenomenon is also considered to be linked with interaction or merging episodes. Sixty galaxies were discarded due to the presence of stellar- or gas-counterrotating components.

\subsection{The final sample}

After exclusion of all the systems belonging to one of the three categories enumerated above, the final sample of normal galaxies includes 1916 objects. The data presented in this catalogue have been collected from the following sources:

- FIR observations, at 60 and $100 \mu \mathrm{m}$ from Knapp et al. (1989) catalogue and the Lyon-Meudon Extragalactic Database (LEDA) (Paturel et al. 1997). These references furnish the bulk of the FIR data used here: 992 detections and upper limits from Knapp et al. (1989) and 711 from LEDA. In the latter case, both the $60 \mu \mathrm{m}$ and $100 \mu \mathrm{m}$ fluxes $\left(\mathrm{S}_{60}\right.$ and $\left.\mathrm{S}_{100}\right)$ are available in the raw data, kindly provided by G. Paturel. Many of the galaxies (346) are also listed in Roberts et al. (1991). Additional IR data for 81 galaxies have been extracted from the NASA/IPAC extragalactic database (NED) and Lavezzi et al. (1999) (30 galaxies).

- $\mathrm{CO}(1-0)$ observations for 175 galaxies have been taken from a long list of published papers. We extracted data also from Roberts et al. (1991) (58) and from the references listed in Casoli et al. (1998), including 299 galaxies studied in previous works.

- HI data, 855 from LEDA (Paturel et al. 1997), 277 from Roberts et al. (1991), and 251 from various sources, including (Casoli et al. 1998) and NED.

- X-ray data from papers based on observations made with the Einstein (Fabbiano et al. 1992; Burstein et al. 1997) and the ROSAT (Beuing et al. 1999) missions. They furnish 290, 683 and 247 values respectively, with many galaxies in common. A more recent revision of these data for 343 galaxies has been presented by O'Sullivan et al. (2001).

For each galaxy we also collected the distance, the blue luminosity and the size. To standardize the information contained in our catalogue we decided to use the morphological classification, distances, sizes and optical luminosities derived from a single source: the LEDA archive. These data were used to normalize the values of FIR and X-ray luminosities as well 


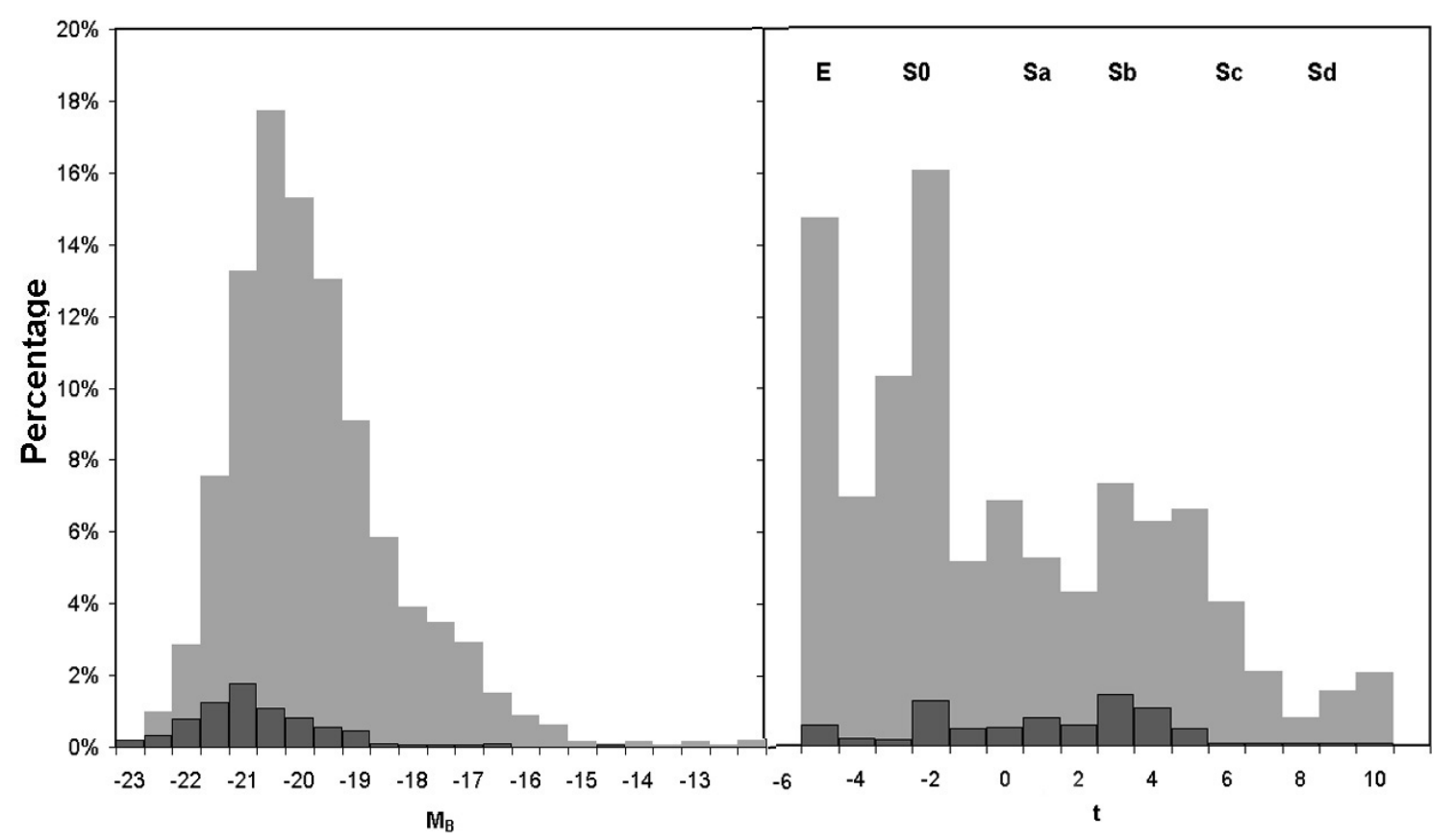

Fig. 1. Luminosity function and distribution according to morphological type derived for the galaxies in the catalogue. Darker histograms represent AGN hosts, while light gray bars include all galaxies in the sample.

as the atomic and molecular gas masses. More precisely, we have extracted the following items for each galaxy: PGC number, morphological type and related numerical parameter $t$, distance modulus and absolute magnitude. The distance moduli are mainly derived from redshifts, corrected for Virgocentric inflow and adopting $H_{\mathrm{o}}=70 \mathrm{~km} \mathrm{~s}^{-1} \mathrm{Mpc}^{-1}$. When redshift was not available, we used the photometric distance modulus, if present in LEDA. Information lacking in LEDA for some galaxies was completed using the ADS bibliographic archive, the NED database or the SIMBAD service of the Strasbourg Centre of Donnée Stellaire (CDS). Finally, the very few galaxies having no classification in the literature were classified by us looking at the optical images extracted from NED.

The luminosity function and the distribution of morphological types are shown in Fig. 1. The distribution of morphological types has two maxima for ellipticals and lenticulars. However, the rest of morphological types are equally represented in the catalogue with similar percentages. Altogether, spirals and irregulars $(t \geq 0)$ represent $40 \%$ of our sample. In Fig. 1 galaxies hosting AGN are represented with dark shaded histograms. At first sight, their mean blue luminosity is slightly above the average value for the whole sample; on the other hand, the morphological type distribution of AGN is more weighted by spiral galaxies compared to the whole sample.

\section{Data processing}

\subsection{Mass estimates}

We have estimated total gas masses and/or luminosities following standard procedures (e.g. see Roberts et al. 1991). The number of detections and upper limits in our sample are listed in Table 2. In the following formulae, the distance $d$ is always in $\mathrm{Mpc}$.
From the value of the IR fluxes at 60 and $100 \mu \mathrm{m}$ we estimated the dust temperature $(\mathrm{K})$ :

$T_{\mathrm{d}}=49 \times\left(S_{60} / S_{100}\right)^{0.4}$.

The corresponding warm dust mass, in solar units, follows from the expression:

$\log M_{\mathrm{d}}=-2.32+\log S_{100}+2 \log d+\log \left(\exp \left(\frac{144.06}{T_{\mathrm{d}}}\right)-1\right)$.

Where $S_{100}$ is in mJy. When data for a single galaxy were available in several catalogues, we compared the mass values and the data quality, and derived accordingly in Table 1 a weighted mean value.

HI gas masses were derived mainly from $21 \mathrm{~cm}$ fluxes $S_{21}$ or from the $m_{21}$ magnitudes of LEDA. From individual papers giving HI masses only, we have scaled the published values to the adopted galaxy distance. From $m_{21}$, we used the expression:

$\log M_{\mathrm{HI}}=5.37-0.4\left(m_{21 c}-17.4\right)+2 \log d$

while if $S_{21}$, in $\mathrm{Jy} \mathrm{km} \mathrm{s}^{-1}$, was available we used the formula:

$\log M_{\mathrm{HI}}=5.37+\log S_{21}+2 \log d$.

Similarly, we have produced mean values when data from different sources were simultaneously available. When both upper limits and detections were available only detections have been used to compute mean values. Moreover, when several upper limits were available, only the lowest value has been adopted.

The data needed to derive the molecular gas content have been taken from various sources (see Appendix A). The formula used to derive molecular gas masses from $\mathrm{CO}(1-0)$ line fluxes $\left(S_{\mathrm{CO}}\right.$ in $\left.\mathrm{Jy} \mathrm{km} \mathrm{s}^{-1}\right)$ is:

$\log M_{\mathrm{mol}}=4.17+2 \log d+\log S_{\mathrm{CO}}$. 
Table 1. An illustration of the catalogue format.

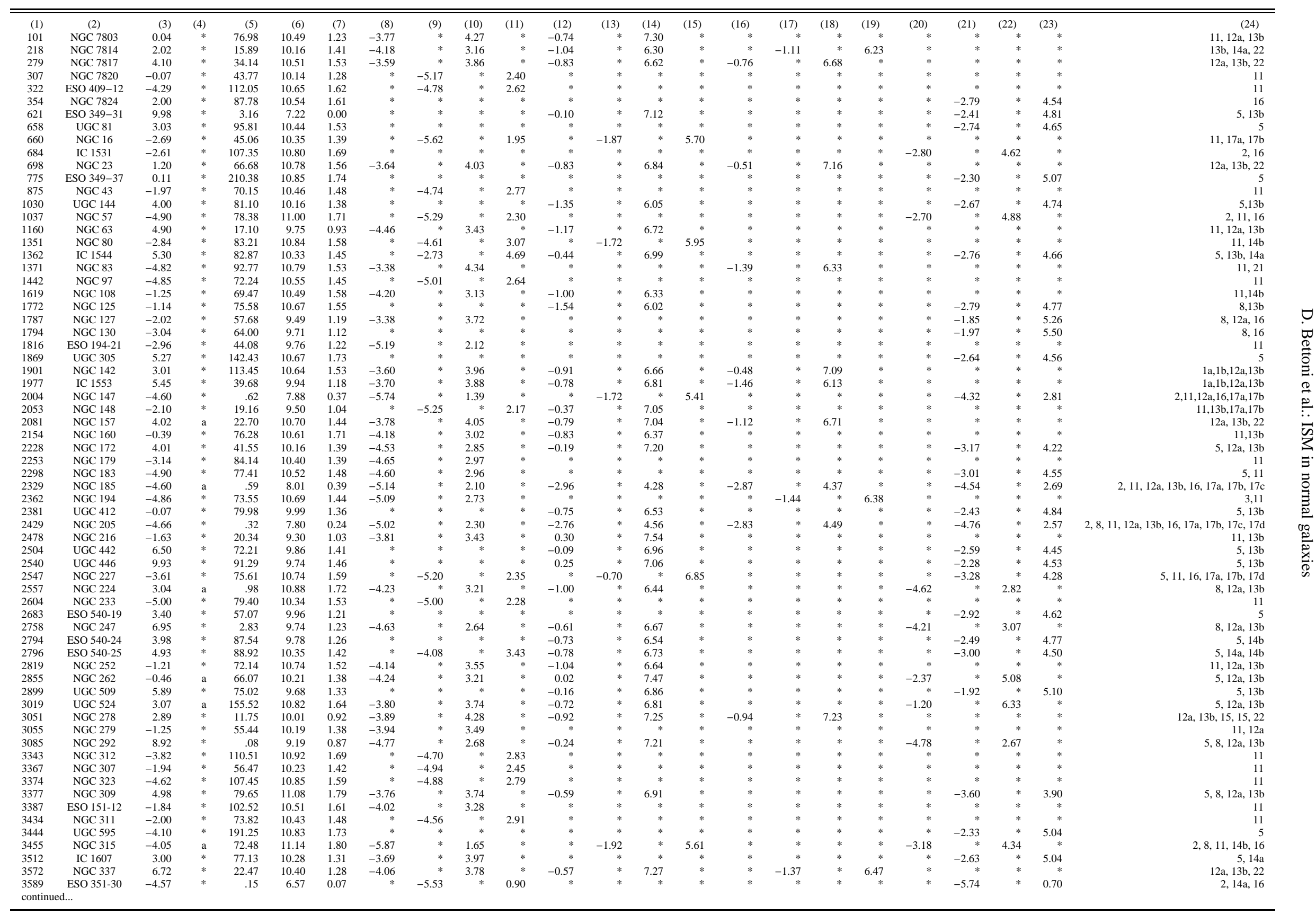


Table 2. Number of detections (Det) and upper limit values $(U L)$ available for the galaxies in Table 1. $N(t)$ represents the total number of galaxies per morphological type bin.

\begin{tabular}{|c|c|c|c|c|c|c|c|c|c|c|}
\hline \multirow[b]{2}{*}{ Type } & \multirow[b]{2}{*}{$t$} & \multirow[t]{2}{*}{$N(t)$} & \multicolumn{2}{|c|}{ Dust } & \multicolumn{2}{|c|}{ HI } & \multicolumn{2}{|c|}{ molecular } & \multicolumn{2}{|c|}{$X$} \\
\hline & & & Det & $\overline{U L}$ & $\overline{D e t}$ & $\overline{U L}$ & $\overline{D e t}$ & $\overline{U L}$ & $\overline{D e t}$ & $\overline{U L}$ \\
\hline E & -5 & 282 & 96 & 162 & 36 & 55 & 10 & 18 & 87 & 73 \\
\hline $\mathrm{E}$ & -4 & 133 & 46 & 72 & 5 & 20 & 2 & 1 & 30 & 34 \\
\hline $\mathrm{E} / \mathrm{S} 0$ & -3 & 197 & 88 & 83 & 19 & 29 & 8 & 3 & 31 & 61 \\
\hline So & -2 & 307 & 154 & 114 & 74 & 60 & 16 & 13 & 32 & 73 \\
\hline SOa & -1 & 98 & 70 & 19 & 41 & 22 & 18 & 1 & 11 & 24 \\
\hline SOa & 0 & 131 & 92 & 14 & 76 & 12 & 17 & 6 & 12 & 27 \\
\hline $\mathrm{Sa}$ & 1 & 100 & 82 & 2 & 67 & 9 & 17 & 9 & 17 & 31 \\
\hline Sab & 2 & 82 & 67 & 1 & 68 & 3 & 29 & 8 & 14 & 36 \\
\hline $\mathrm{Sb}$ & 3 & 140 & 113 & 4 & 117 & 3 & 53 & 7 & 24 & 60 \\
\hline $\mathrm{Sbc}$ & 4 & 120 & 90 & 5 & 103 & 2 & 48 & 4 & 18 & 63 \\
\hline $\mathrm{Sc}$ & 5 & 126 & 98 & 5 & 105 & 3 & 58 & 7 & 23 & 50 \\
\hline $\mathrm{Sc}$ & 6 & 77 & 48 & 1 & 73 & 0 & 20 & 11 & 7 & 50 \\
\hline Scd & 7 & 40 & 25 & 0 & 37 & 1 & 9 & 13 & 3 & 19 \\
\hline $\mathrm{Sd}$ & 8 & 15 & 8 & 0 & 13 & 0 & 3 & 2 & 2 & 8 \\
\hline $\mathrm{Sm}$ & 9 & 29 & 13 & 0 & 23 & 0 & 3 & 1 & 4 & 20 \\
\hline Irr & 10 & 39 & 14 & 1 & 34 & 1 & 6 & 6 & 4 & 25 \\
\hline
\end{tabular}

We implicitly assume a constant $\mathrm{CO} / \mathrm{H}_{2}$ conversion factor $\chi=$ $N\left(\mathrm{H}_{2}\right) / I_{\mathrm{CO}}=2.3 \times 10^{20} \mathrm{~mol} / \mathrm{K} \mathrm{km} \mathrm{s}^{-1}$ (Strong 1988); $M_{\mathrm{mol}}$ includes the helium mass fraction by multiplying the $\mathrm{H}_{2}$ mass by 1.36 . When only mass values were available in the consulted references, these have been scaled to the distances assumed here. As discussed in Paper I, a source of uncertainty for the estimates of $M_{\mathrm{mol}}$ comes from the undersampling of some of the sources mapped in $\mathrm{CO}$. However the majority of $\mathrm{CO}$ observations considered here sample fairly well the emission in the inner galaxy disks. As we expect the bulk of $\mathrm{CO}$ emission to come precisely from these regions, the derived $M_{\mathrm{mol}}$ should be taken as a meaningful lower limit for the total molecular gas mass. A variation of the $\mathrm{CO}-\mathrm{H}_{2}$ conversion factor $-\chi-$ can neither be excluded and may be caused by the different metallicities or physical conditions for molecular gas among the galaxies in the sample. Before any further improvement can be envisaged we will assume the above derived values as a good estimate of the molecular gas masses.

$\mathrm{X}$-ray emission in galaxies may derive from diffuse gas and/or from discrete sources. This means that the mass estimate of hot gas strongly depends on the prevalent mechanism at work in a particular galaxy. Hence, mass estimates cannot be described by a simple formula. We thus decided to use the X-ray luminosities, instead of the masses, to describe the canonical X-ray yield for galaxies in our sample. A discussion of the differences existing in literature data on X-ray observations with different telescopes has been made by Beuing et al. (1999).

We have 325 detections and 626 upper limits among the inactive galaxies; and 84 detections and 29 upper limits from galaxies indicated in the Veron et al. (2000) catalogue as AGN (139) or containing quasars (6) or BL Lac (3).
To avoid zero-order biases we have normalized the gas masses and luminosities using two different normalization factors: the total blue luminosity $-L_{\mathrm{B}}-$ and the square of the linear diameter measured at $25 \mathrm{mag} \operatorname{arcsec}^{-2}-D_{25}^{2}$. These parameters are available for all the 1916 galaxies of our sample. The absolute blue magnitudes (from LEDA catalogue) are corrected for dust absorption and cosmological reddening. The global correction is also a function of the type (Paturel et al. 1997); the correction factor increases toward later types.

The mean value of $\log L_{\mathrm{B}} / D_{25}^{2}$, derived for the galaxies in our sample is:

$\log L_{\mathrm{B}} / D_{25}^{2}=-0.013 t+7.6$

with $L_{\mathrm{B}} / D_{25}^{2}$ in $L_{\odot} / \mathrm{kpc}^{2}$ with an rms of $\pm 0.3(1 \sigma)$. As we shall see in the following paragraphs, this relatively low slope produces similar trends in the $M / L_{\mathrm{B}}$ and $M / D_{25}^{2}$ mean ratios derived in this work.

\subsection{Stability of the results}

In principle, $M / L_{\mathrm{B}}$ and $M / D_{25}^{2}$ ratios do not depend at all on the adopted distance of the galaxy. This makes our results insensitive to the specific value of the Hubble constant adopted. However, distances and magnitudes are affected differently by the corrections for the Virgocentric motion and by the galactic and internal absorptions. Hence, $M / L_{\mathrm{B}}$ and $M / D_{25}^{2}$ may change significantly. Since the first approach to this catalogue was published by Bettoni et al. (2001), the LEDA database has been modified, because the stored data are continuously being updated. In addition to these changes, a significant amount of new data from other sources have been included in the new catalogue.

While these changes forced us to recalculate all the ratios, this allowed us to test the stability of our results. Comparing the data extracted from this database in different dates in the last two years, we found that the $90 \%$ of the $\log M / L_{\mathrm{B}}$ and the $98 \%$ of the $\log M / D_{25}^{2}$ values calculated from these different data extractions differ by less than $1 \sigma$, with no variation larger than $2 \sigma$. Within these ranges we expect our conclusions to be stable.

\subsection{Derivation of mean ratios: $\log M / D^{2}-\log M / L$}

In order to define the mean content of each tracer according to the type, we made use of survival analysis methods (see Feigelson \& Nelson 1999), applied to the different ensembles of $M / L_{\mathrm{B}}$ and $M / D_{25}^{2}$ data. This analysis tool properly takes into account both detections and upper limits (UL) in order to derive representative averages. The mean values are derived and shown in the following Tables, binned according to the morphological type code. This method also allows a more direct comparison of our data with that from literature.

Survival analysis (Feigelson \& Nelson 1999) programs are part of several packages of data analysis, such as IRAF, and may be applied when a number of upper or lower limit values are available together with values from detections. Making use of the Kaplan-Meier estimator, we derived, for each $t$, 
Table 3. Mean warm dust content according to morphological type. $N$ represents the total number of galaxies which have data (detections and upper limits), while $N_{\mathrm{ds}}$ indicates the number of detections resulting from the survival analysis statistics. When upper limits exist, mean values are derived making use of survival analysis. All values are normalized to $L_{\mathrm{B}}$, the corrected blue luminosities, and to $D_{25}^{2}$, the square of the diameter in $\mathrm{kpc}$ at the isophote of $25 \mathrm{mag} \operatorname{arcsec}^{-2}$.

\begin{tabular}{lrrrr}
\hline \hline Type & $t$ & $N / N_{\mathrm{ds}}$ & $M_{\text {dust }} / L_{\mathrm{B}}$ & $M_{\text {dust }} / D_{25}^{2}$ \\
& & & & \\
\hline $\mathrm{E}$ & -5 & $259 / 98$ & $-5.99 \pm 0.12$ & $1.53 \pm 0.13$ \\
$\mathrm{E}$ & -4 & $118 / 47$ & $-5.60 \pm 0.13$ & $1.94 \pm 0.14$ \\
$\mathrm{E} / \mathrm{S} 0$ & -3 & $171 / 89$ & $-5.19 \pm 0.09$ & $2.42 \pm 0.09$ \\
$\mathrm{~S} 0$ & -2 & $269 / 156$ & $-4.97 \pm 0.07$ & $2.51 \pm 0.08$ \\
$\mathrm{~S} 0 \mathrm{a}$ & -1 & $87 / 69$ & $-4.63 \pm 0.13$ & $2.79 \pm 0.12$ \\
$\mathrm{~S} 0 \mathrm{a}$ & 0 & $108 / 95$ & $-4.34 \pm 0.08$ & $3.10 \pm 0.08$ \\
$\mathrm{Sa}$ & 1 & $82 / 80$ & $-4.07 \pm 0.06$ & $3.49 \pm 0.06$ \\
$\mathrm{Sab}$ & 2 & $68 / 67$ & $-3.82 \pm 0.05$ & $3.70 \pm 0.05$ \\
$\mathrm{Sb}$ & 3 & $117 / 113$ & $-3.75 \pm 0.04$ & $3.83 \pm 0.05$ \\
$\mathrm{Sbc}$ & 4 & $96 / 91$ & $-3.74 \pm 0.03$ & $3.83 \pm 0.04$ \\
$\mathrm{Sc}$ & 5 & $102 / 97$ & $-3.78 \pm 0.03$ & $3.82 \pm 0.04$ \\
$\mathrm{Sc}$ & 6 & $50 / 49$ & $-3.92 \pm 0.05$ & $3.58 \pm 0.07$ \\
$\mathrm{Scd}$ & 7 & $24 / 24$ & $-4.09 \pm 0.07$ & $3.34 \pm 0.09$ \\
$\mathrm{Sd}$ & 8 & $8 / 8$ & $-4.50 \pm 0.23$ & $3.13 \pm 0.24$ \\
$\mathrm{Sm}$ & 9 & $13 / 13$ & $-4.40 \pm 0.21$ & $3.04 \pm 0.24$ \\
$\mathrm{Irr}$ & 10 & $15 / 14$ & $-4.12 \pm 0.16$ & $3.43 \pm 0.19$ \\
& & & & \\
\hline Total & & $1587 / 1110$ & &
\end{tabular}

mean values of mass/light and mass/area ratios and the corresponding standard deviations. When all the galaxies considered within an interval of $t$ are detected, these values coincide with that from arithmetic means. On the contrary, the method cannot be applied when both upper and lower limits are present, for instance when considering the mass ratios (e.g. $M_{\mathrm{mol}} / M_{\mathrm{HI}}$ ). In such a case, we present ratios of mean values.

\subsection{Description of the full version of the catalogue}

All the data of this catalogue are listed in Table 1, which is only available in digital form. The first page is shown in Table 1 of this paper, as an illustration of the on-line catalogue format. The columns are: (1) the PGC identifier and (2) the galaxy name, (3) the numerical type code $t$ (de Vaucouleurs et al. 1991; Paturel et al. 1997), (4) this column indicates the presence of nuclear activity (Veron et al. 2000) with the letter "a", (5) the adopted distance $d$ (in Mpc), (6) the logarithm of the total blue luminosity $L_{\mathrm{B}}$ in solar units, (7) the logarithm of the linear size $D_{25}$ in kpc corresponding to the $25 \mathrm{mag} \mathrm{arcsec}^{-2}$ isophote. The values for each tracer (dust, HI, molecular gas, $\mathrm{X}$-ray) span four columns: the first two are mass vs. $L_{\mathrm{B}}$ and the next two are mass vs. area, defined as $D_{25}^{2}$. Values corresponding to detections of a galaxy in the corresponding tracers are in the first and in the third columns while upper limits are in the second and fourth columns. All masses and luminosities are in solar units. Following this scheme, the following columns contain: $\log M_{\text {dust }} / L_{\mathrm{B}}$ detections (Col. 8) and upper limits (Col. 9), Log $M_{\text {dust }} / D_{25}^{2}$ detections (Col. 10) and upper
Table 4. Mean HI content according to morphological type. Notation as in Table 3.

\begin{tabular}{lrrrc}
\hline \hline Type & $t$ & $N / N_{\mathrm{ds}}$ & $M_{\mathrm{HI}} / L_{\mathrm{B}}$ & $M_{\mathrm{HI}} / D_{25}^{2}$ \\
& & & & \\
\hline $\mathrm{E}$ & -5 & $91 / 37$ & $-2.34 \pm 0.14$ & $5.33 \pm 0.13$ \\
$\mathrm{E}$ & -4 & $25 / 6$ & $-1.83 \pm 0.12$ & $5.54 \pm 0.14$ \\
$\mathrm{E} / \mathrm{S} 0$ & -3 & $49 / 20$ & $-1.89 \pm 0.16$ & $5.70 \pm 0.15$ \\
$\mathrm{~S} 0$ & -2 & $134 / 75$ & $-1.55 \pm 0.09$ & $5.96 \pm 0.09$ \\
$\mathrm{~S} 0 \mathrm{a}$ & -1 & $62 / 41$ & $-1.81 \pm 0.19$ & $5.58 \pm 0.19$ \\
$\mathrm{~S} 0 \mathrm{a}$ & 0 & $88 / 77$ & $-0.96 \pm 0.06$ & $6.47 \pm 0.06$ \\
$\mathrm{Sa}$ & 1 & $76 / 67$ & $-1.05 \pm 0.07$ & $6.52 \pm 0.07$ \\
$\mathrm{Sab}$ & 2 & $71 / 68$ & $-0.95 \pm 0.06$ & $6.57 \pm 0.06$ \\
$\mathrm{Sb}$ & 3 & $120 / 117$ & $-0.77 \pm 0.04$ & $6.78 \pm 0.03$ \\
$\mathrm{Sbc}$ & 4 & $106 / 104$ & $-0.66 \pm 0.03$ & $6.90 \pm 0.03$ \\
$\mathrm{Sc}$ & 5 & $107 / 104$ & $-0.67 \pm 0.04$ & $6.92 \pm 0.03$ \\
$\mathrm{Sc}$ & 6 & $74 / 74$ & $-0.48 \pm 0.04$ & $6.93 \pm 0.04$ \\
$\mathrm{Scd}$ & 7 & $37 / 36$ & $-0.33 \pm 0.07$ & $6.96 \pm 0.05$ \\
$\mathrm{Sd}$ & 8 & $13 / 13$ & $-0.32 \pm 0.19$ & $7.11 \pm 0.11$ \\
$\mathrm{Sm}$ & 9 & $23 / 23$ & $-0.29 \pm 0.08$ & $7.02 \pm 0.06$ \\
$\mathrm{Irr}$ & 10 & $35 / 34$ & $-0.20 \pm 0.10$ & $7.05 \pm 0.06$ \\
& & & & \\
\hline Total & & $1111 / 896$ & &
\end{tabular}

limits (Col. 11), $\log M_{\mathrm{HI}} / L_{\mathrm{B}}$ detections (Col. 12) and upper limits (Col. 13), Log $M_{\mathrm{HI}} / D_{25}^{2}$ detections (Col. 14) and upper limits (Col. 15), $\log M_{\mathrm{mol}} / L_{\mathrm{B}}$ detections (Col. 16) and upper limits (Col. 17), $\log M_{\mathrm{mol}} / D_{25}^{2}$ detections (Col. 18) and upper limits (Col. 19), $\log L_{\mathrm{X}} / L_{\mathrm{B}}$ detections (Col. 20) and upper limits (Col. 21), Log $L_{\mathrm{X}} / D_{25}^{2}$ detections (Col. 22) and upper limits (Col. 23). References for the gas masses are listed in the last column (24). The reference codes are given in the Appendix.

This table contains all the essential information compiled for this catalogue. This compilation is intended to serve as a template for future studies on the gas content in selected types of galaxies.

\section{Analysis of main results}

Tables 2-6 give the normalized mean gas contents $-\log M / D^{2}$ and $\log M / L_{\mathrm{B}}-$ and the detection rates for the galaxies of the sample, as a function of the Hubble type. The galaxies are identified according to the numerical code $t$ (RC3 morphological type). Some types have two numerical codes: elliptical galaxies span the types -5 and -4 ; S0-a the types -1 and 0 and Sc galaxies fill the types 5 and 6 ; all the remaining galaxy types are identified by a single code. Note that the code 5.00 also includes spiral galaxies with ambiguous classification (type $\mathrm{S}$ ?). In our sample there are only 7 galaxies falling into this category.

\subsection{Detection rates}

As far as detection rates are concerned, we have found the following trends:

- the detection rate of elliptical galaxies in X-ray wavelengths from our data is 1.3-4 times higher than that of 
Table 5. Mean content of molecular gas according to morphological type. Notation as in Table 3. The values for $t=-4$ and $t=8$ are based on a low number of galaxies and are more uncertain.

\begin{tabular}{lrrrr}
\hline \hline Type & $t$ & $N / N_{\mathrm{ds}}$ & $M_{\mathrm{mol}} / L_{\mathrm{B}}$ & $M_{\mathrm{mol}} / D_{25}^{2}$ \\
& & & & \\
\hline $\mathrm{E}$ & -5 & $28 / 11$ & $-2.53 \pm 0.22$ & $5.03 \pm 0.22$ \\
$\mathrm{E}$ & -4 & $3 / 3$ & $-1.25 \pm 0.23$ & $6.34 \pm 0.22$ \\
$\mathrm{E} / \mathrm{S} 0$ & -3 & $11 / 8$ & $-2.23 \pm 0.32$ & $5.33 \pm 0.33$ \\
$\mathrm{~S} 0$ & -2 & $30 / 17$ & $-1.82 \pm 0.21$ & $5.76 \pm 0.22$ \\
$\mathrm{~S} 0 \mathrm{a}$ & -1 & $18 / 18$ & $-1.41 \pm 0.19$ & $6.00 \pm 0.17$ \\
$\mathrm{~S} 0 \mathrm{a}$ & 0 & $24 / 18$ & $-1.14 \pm 0.15$ & $6.33 \pm 0.18$ \\
$\mathrm{Sa}$ & 1 & $25 / 18$ & $-1.13 \pm 0.17$ & $6.52 \pm 0.16$ \\
$\mathrm{Sab}$ & 2 & $37 / 30$ & $-1.17 \pm 0.13$ & $6.39 \pm 0.16$ \\
$\mathrm{Sb}$ & 3 & $60 / 53$ & $-0.96 \pm 0.08$ & $6.63 \pm 0.08$ \\
$\mathrm{Sbc}$ & 4 & $53 / 49$ & $-0.88 \pm 0.06$ & $6.75 \pm 0.07$ \\
$\mathrm{Sc}$ & 5 & $64 / 58$ & $-1.01 \pm 0.06$ & $6.58 \pm 0.07$ \\
$\mathrm{Sc}$ & 6 & $31 / 20$ & $-1.44 \pm 0.11$ & $5.99 \pm 0.14$ \\
$\mathrm{Scd}$ & 7 & $22 / 9$ & $-1.92 \pm 0.16$ & $5.54 \pm 0.18$ \\
$\mathrm{Sd}$ & 8 & $5 / 3$ & $-1.52 \pm 0.35$ & $6.28 \pm 0.34$ \\
$\mathrm{Sm}$ & 9 & $4 / 4$ & $-1.54 \pm 0.32$ & $6.05 \pm 0.49$ \\
$\mathrm{Irr}$ & 10 & $12 / 6$ & $-1.71 \pm 0.28$ & $5.90 \pm 0.35$ \\
& & & & \\
\hline Total & & $427 / 325$ & & \\
\end{tabular}

late-type galaxies. As expected, the percentage of X-ray detections for spirals increases by $10 \%$ if AGN galaxies are included in the sample;

- on the other hand, compared with ellipticals, both CO and HI detections are $\sim 4$ times more common in disk galaxies (note however that $\sim 40 \%$ of type $t=-5$ ellipticals have been detected in $\mathrm{CO}$ or $\mathrm{HI})$. This high detection rate in spirals reflects the higher cold gas content of disk galaxies. The dust detection roughly follows the HI detection rates.

\subsection{Comparison with previous work}

Bregman et al. (1992) and Casoli et al. (1998) have previously addressed the study of the ISM content in galaxies and its variation along the Hubble sequence. Both groups based their study on two samples of 467 (Bregman et al. 1992) and 582 (Casoli et al. 1998) galaxies, compiled and processed according to different criteria. Our catalogue improves by a significant factor ( 3-4) the overall statistics of previous samples. The most significant improvement corresponds to the left branch of the Hubble sequence: compared to previous works, the number of early-type galaxies $(t<0)$ with firm detections processed for this catalogue is $>3$ times larger.

Bregman et al. (1992) analyzed their sample using other non-parametric tests, based on rank, to study the variation of the mean gas content with morphological type. The estimated averages were normalized to the galaxy blue luminosity. Their results indicate that the mean content of atomic gas (in $M / L_{\mathrm{B}}$ scale) decreases by two orders of magnitude from early-type spirals (Sa) to ellipticals $(\mathrm{E})$. A similar result is obtained for the mean content of molecular gas. Altogether, these results underlined that ellipticals have a neutral gas
Table 6. Mean X-ray luminosity of the galaxy $L_{X}$ versus the morphological type $t$. All values are normalized to $L_{\mathrm{B}}$, the corrected blue luminosities, or $D_{25}^{2}$, the square of the diameter in $\mathrm{kpc}$ at the isophote of $25 \mathrm{mag} \operatorname{arcsec}^{-2}$. The values of the first table were calculated excluding the galaxies hosting AGN. The second table includes them.

\begin{tabular}{|c|c|c|c|c|}
\hline \multicolumn{5}{|c|}{ Without AGN } \\
\hline Type & $t$ & $N / N_{\mathrm{ds}}$ & $\log L_{\mathrm{Xt}} / L_{\mathrm{B}}$ & $\log L_{\mathrm{Xt}_{\mathrm{t}}} / D_{25}^{2}$ \\
\hline $\mathrm{E}$ & -5 & $150 / 79$ & $-3.69 \pm 0.122$ & $3.62 \pm 0.19$ \\
\hline $\mathrm{E}$ & -4 & $61 / 29$ & $-3.42 \pm 0.14$ & $4.08 \pm 0.15$ \\
\hline $\mathrm{E} / \mathrm{S} 0$ & -3 & $92 / 31$ & $-3.97 \pm 0.22$ & $3.72 \pm 0.16$ \\
\hline So & -2 & $91 / 24$ & $-3.78 \pm 0.10$ & $3.61 \pm 0.11$ \\
\hline SOa & -1 & $28 / 7$ & $-3.82 \pm 0.07$ & $3.68 \pm 0.09$ \\
\hline SOa & 0 & $31 / 4$ & $-3.67 \pm 0.08$ & $3.69 \pm 0.11$ \\
\hline $\mathrm{Sa}$ & 1 & $38 / 8$ & $-4.03 \pm 0.32$ & $3.75 \pm 0.15$ \\
\hline $\mathrm{Sab}$ & 2 & $40 / 7$ & $-3.99 \pm 0.14$ & $3.47 \pm 0.12$ \\
\hline $\mathrm{Sb}$ & 3 & $63 / 10$ & $-4.24 \pm 0.15$ & $3.32 \pm 0.13$ \\
\hline $\mathrm{Sbc}$ & 4 & $65 / 8$ & $-4.01 \pm 0.08$ & $3.49 \pm 0.09$ \\
\hline $\mathrm{Sc}$ & 5 & $65 / 19$ & $-4.04 \pm 0.09$ & $3.50 \pm 0.11$ \\
\hline $\mathrm{Sc}$ & 6 & $57 / 7$ & $-4.29 \pm 0.12$ & $3.01 \pm 0.17$ \\
\hline Scd & 7 & $20 / 2$ & $-4.26 \pm 0.03$ & $3.16 \pm 0.08$ \\
\hline $\mathrm{Sd}$ & 8 & $9 / 1$ & $-5.17 \pm 0.03$ & $1.83 \pm 0.00$ \\
\hline $\mathrm{Sm}$ & 9 & $23 / 4$ & $-4.22 \pm 0.28$ & $3.06 \pm 0.29$ \\
\hline Irr & 10 & $28 / 3$ & $-3.85 \pm 0.23$ & $3.43 \pm 0.17$ \\
\hline Total & \multicolumn{3}{|c|}{$861 / 243$} & \\
\hline \multicolumn{5}{|c|}{ With AGN } \\
\hline Type & $t$ & $N / N_{\mathrm{ds}}$ & $\log L_{\mathrm{Xt}} / L_{\mathrm{B}}$ & $\log L_{\mathrm{Xt}} / D_{25}^{2}$ \\
\hline Total & \multicolumn{3}{|c|}{$945 / 280$} & \\
\hline E & -5 & $161 / 88$ & $-3.65 \pm 0.12$ & $3.65 \pm 0.18$ \\
\hline E & -4 & $63 / 31$ & $-3.36 \pm 0.14$ & $4.15 \pm 0.15$ \\
\hline $\mathrm{E} / \mathrm{S} 0$ & -3 & $94 / 32$ & $-4.03 \pm 0.22$ & $3.69 \pm 0.16$ \\
\hline S0 & -2 & $104 / 33$ & $-3.59 \pm 0.11$ & $3.82 \pm 0.12$ \\
\hline $\mathrm{S} 0 / \mathrm{a}$ & -1 & $35 / 11$ & $-3.60 \pm 0.15$ & $3.89 \pm 0.16$ \\
\hline S0/a & 0 & $39 / 12$ & $-3.30 \pm 0.14$ & $4.11 \pm 0.16$ \\
\hline $\mathrm{Sa}$ & 1 & $48 / 17$ & $-3.69 \pm 0.25$ & $4.09 \pm 0.17$ \\
\hline Sab & 2 & $50 / 15$ & $-3.79 \pm 0.13$ & $3.72 \pm 0.12$ \\
\hline $\mathrm{Sb}$ & 3 & $84 / 25$ & $-3.98 \pm 0.12$ & $3.57 \pm 0.12$ \\
\hline $\mathrm{Sbc}$ & 4 & $81 / 18$ & $-3.82 \pm 0.09$ & $3.69 \pm 0.09$ \\
\hline $\mathrm{Sc}$ & 5 & $73 / 24$ & $-3.97 \pm 0.09$ & $3.57 \pm 0.10$ \\
\hline $\mathrm{Sc}$ & 6 & $58 / 7$ & $-4.29 \pm 0.12$ & $3.01 \pm 0.17$ \\
\hline Scd & 7 & $21 / 3$ & $-4.20 \pm 0.06$ & $3.23 \pm 0.09$ \\
\hline $\mathrm{Sd}$ & 8 & $10 / 2$ & $-4.91 \pm 0.18$ & $2.60 \pm 0.54$ \\
\hline $\mathrm{Sm}$ & 9 & $24 / 4$ & $-4.28 \pm 0.24$ & $3.01 \pm 0.26$ \\
\hline Irr & 10 & $29 / 4$ & $-3.88 \pm 0.17$ & $3.40 \pm 0.15$ \\
\hline
\end{tabular}

Total

$974 / 326$

content significantly lower than lenticulars (see Fig. 2). The sample of Bregman et al. (1992) has only 7 (35) firm detections in $\mathrm{CO}(\mathrm{HI})$ for galaxies spanning the lenticular-elliptical range.

The scenario depicted by Casoli et al. (1998) seems at odds with Bregman et al. (1992)'s results, however. Casoli et al. (1998) used a survival analysis method and normalized their data by $D_{25}^{2}$. According to Casoli et al. (1998), the atomic mean gas content shows little variation going from elliptical to lenticular galaxies (see upper panel of Fig. 2). Within the same type range, the molecular gas content varies by less than one order of 

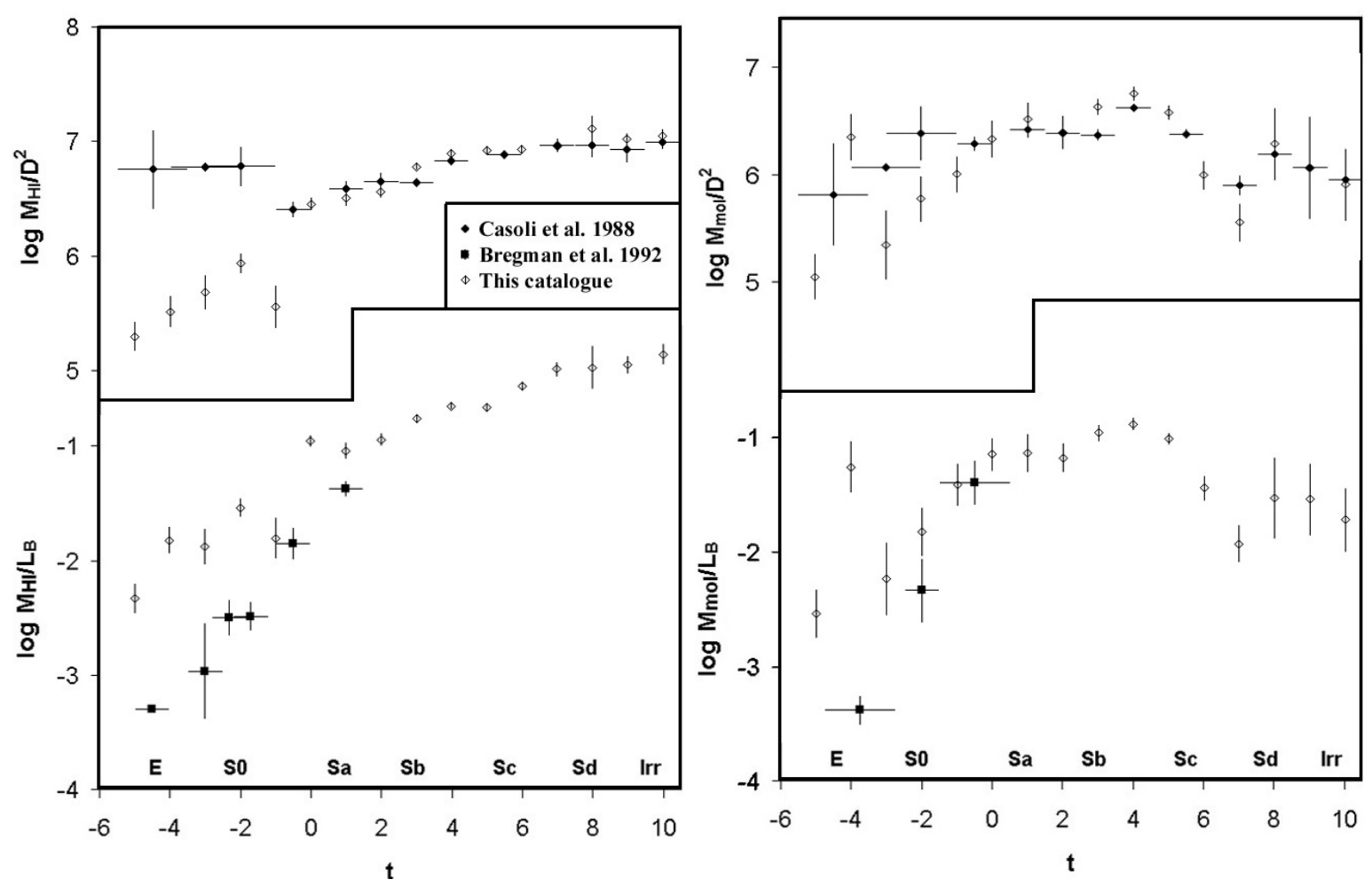

Fig. 2. Comparison between the mean $\log M / L$ ratios for HI (left panels) and for molecular gas (right panels) extracted from the present catalogue and mean $\log M / L$ ratios of previous studies (Bregman et al. 1992; Casoli et al. 1998). In the upper panel the mean masses are normalized with respect to $D_{25}^{2}$, as adopted by Casoli et al. (1998), while in the lower panel $L_{\mathrm{B}}$ is the normalization factor, as adopted by Bregman et al. (1992). Our estimates agree with those published by Casoli et al. (1998) only for types later than S0.

magnitude: i.e., significantly less than shown by Bregman et al. (1992). Casoli et al. (1998) excluded from their sample galaxies in cluster environments, tagged as HI-deficient. This might explain why, in contrast to Bregman et al. (1992), Casoli et al. (1998) find no significant trend for the HI content. The sample of Casoli et al. (1998) has 11 (13) firm detections in CO (HI) for galaxies spanning the lenticular-elliptical range.

These two scenarios might only be reconciled if the mean $L_{\mathrm{B}} / D_{25}^{2}$ ratios were seen to strongly depend on morphological type in the "conflicting" range: ellipticals would need to be much fainter, by at least two orders of magnitude, with respect to S0 of comparable sizes. As it has been discussed in Sect. 3.1, the estimated trend of $\log L_{\mathrm{B}} / D_{25}^{2}$ is much smoother, however; this is confirmed by the similar trends obtained using either $M / L_{\mathrm{B}}$ or $M / D_{25}^{2}$ as gas content indicators.

The strong disagreement between these two studies should be attributable to their poor statistics. This is probably the reason why our estimates of the neutral gas content match neither of previous claims in this range. As it is shown in Table 2, the present catalogue is based on $31 \mathrm{CO}$ detections and $132 \mathrm{HI}$ detections within the range of early type galaxies (ellipticals to lenticulars). Our results, normalized with respect to $L_{\mathrm{B}}$ and $D_{25}^{2}$ both indicate a similar trend: a moderate one order of magnitude decrease of the mean neutral gas content (atomic and/or molecular) going from S0 to E galaxies.

It is worth noting that, in contrast to Casoli et al. (1998), we found that the $M_{\mathrm{mol}} / M_{\mathrm{HI}}$ ratio is roughly constant along the Hubble sequence, except for the latest type systems (Sd and Irr) where this ratio decreases by one order of magnitude. Earlytype galaxies and early spirals possess $\sim 40 \%$ of their neutral gas content in the molecular phase, while late-type spirals and irregulars have $\sim 90 \%$ of their neutral gas content in atomic form. In Fig. 3, we plotted our data together with values derived by Casoli et al. (1998).

\subsection{AGN versus non-active galaxies}

We have found no significant differences in the neutral gas content between galaxies hosting AGNs and those considered as non-active. For this reason, the mean data in Tables 3-4 do not distinguish between active and inactive galaxies, even if it is possible to identify both categories of galaxies in our catalogue.

In our database there are 20 active galaxies that are more luminous than $\log L_{\mathrm{X}} / L_{\mathrm{B}}=-2$, while most of the remaining galaxies have values between -2 and -4 . Apart from the overluminous galaxies, the mean ratios $L_{X} / L_{\mathrm{B}}$ and $L_{\mathrm{X}} / D_{25}^{2}$ show an almost flat trend going from elliptical galaxies to $\mathrm{Sa}$ and a slowly decreasing trend for later types (Fig. 4). In Table 6 we publish the mean values obtained by both including and excluding AGN.

\section{On-line access}

We have presented a catalogue devoted to a study of the ISM content in a sample of normal galaxies. The catalogue has been compiled based on previous catalogues with the 


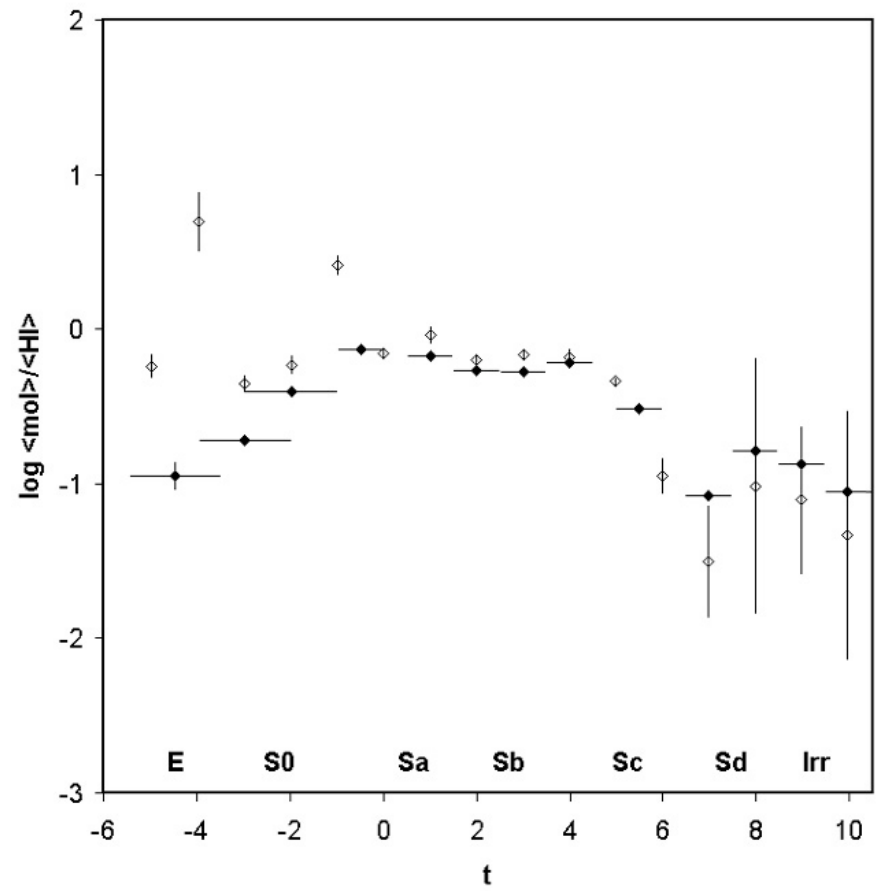

Fig. 3. The mean molecular to atomic gas content ratio as a function of Hubble type. The open symbols are derived from our catalogue, while full symbols represent ratios published by Casoli et al. (1998, full symbols).

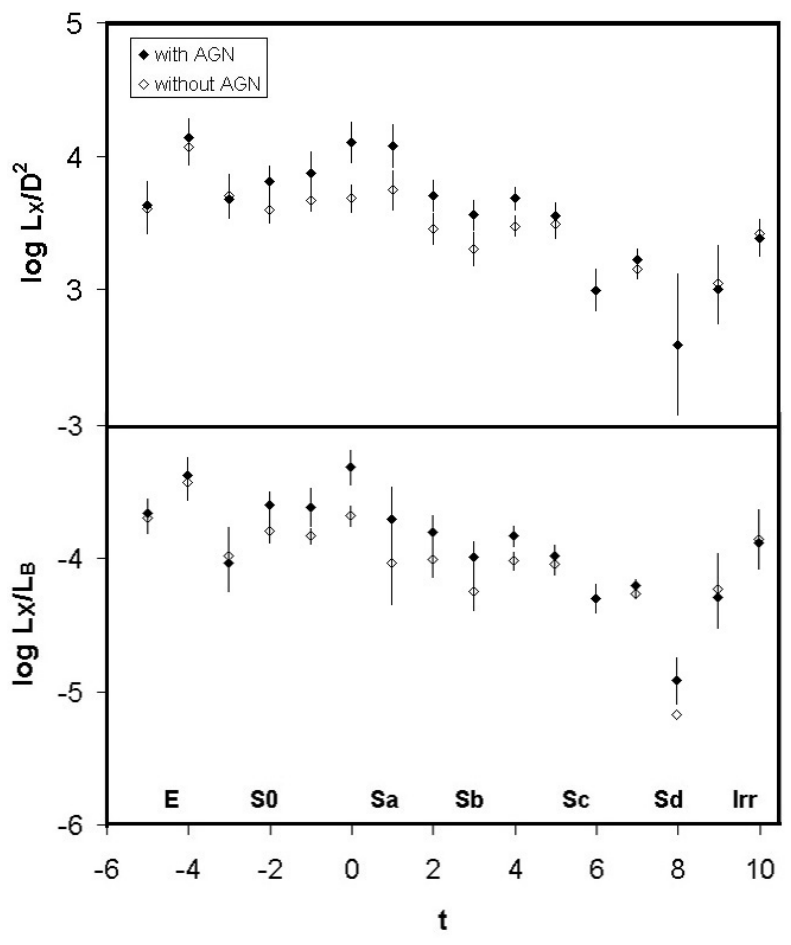

Fig. 4. Variation of mean $\log L_{\mathrm{X}} / D_{25}^{2}$ and $\log L_{\mathrm{X}} / L_{\mathrm{B}}$ ratios either including or excluding AGN host galaxies.

addition of a significant volume of new data. This is intended to serve as a reference defining the canonical gas content of "normal" galaxies along the Hubble sequence. The latest version of the catalogue is also available at http://dipastro.pd.astro.it/galletta/ismcat/.
The number of galaxies included, 1916 systems, is 3-4 times greater than previous studies, and fills a gap currently existing in the ISM studies of cold gas for early-type galaxies. All the catalogue data have been homogenized and reduced to the same distance scale and can be accessed as a single text list, formatted in columns, or consulted at CDS. Upon request, it can be obtained from the authors as an Excel-formatted database.

Acknowledgements. The authors thanks Dr. D. Hogg for the useful comments to this work.

This research made use of Vizier service (Ochsenbein et al. 2000), the SIMBAD database (operated at CDS, Strasbourg, France), the NASA/IPAC Extragalactic Database (NED) (which is operated by JPL, California Institute of Technology, under contract to NASA) and NASA's Astrophysics Data System Abstract Service (mirrored in CDS of Strasbourg). The authors would like to thank Dr. G. Paturel for kindly making the LEDA database's FIR raw data available and Dr. F. Ochsenbein for the changes made to the Vizier's query form on our request. GG made use of funds from the University of Padova (Fondi 60\%-2002).

\section{Appendix A: References to Table 1}

\begin{tabular}{lll}
\hline \hline $\begin{array}{l}\text { Reference } \\
\text { code }\end{array}$ & Source of data & ISM \\
& tracer \\
\hline $1 \mathrm{a}$ & Andreani et al. (1995) & $\mathrm{HI}$ \\
$1 \mathrm{~b}$ & Andreani et al. (1995) & $\mathrm{CO}$ \\
2 & Beuing et al. (1999) & $\mathrm{X}$ \\
3 & Boselli et al. (1995) & $\mathrm{CO}$ \\
4 & Bregman et al. (1992) & $\mathrm{CO}$ \\
5 & Burstein et al. (1997) & $\mathrm{X}$ \\
$6 \mathrm{a}$ & Casoli et al. (1991) & $\mathrm{HI}$ \\
$6 \mathrm{~b}$ & Casoli et al. (1991) & $\mathrm{CO}$ \\
7 & Casoli et al. (1998) & $\mathrm{CO}$ \\
8 & Fabbiano et al. (1992) & $\mathrm{X}$ \\
9 & Gerin \& Casoli (1994) & $\mathrm{CO}$ \\
$10 \mathrm{a}$ & Horellou et al. (1995) & $\mathrm{HI}$ \\
$10 \mathrm{~b}$ & Horellou et al. (1995) & $\mathrm{CO}$ \\
11 & Knapp et al. (1989) & $\mathrm{IR}$ \\
$12 \mathrm{a}$ & Lavezzi et al. (1999) & $\mathrm{IR}$ \\
$12 \mathrm{~b}$ & Lavezzi et al. (1999) & $\mathrm{HI}$ \\
$12 \mathrm{c}$ & Lavezzi et al. (1999) & $\mathrm{CO}$ \\
$13 \mathrm{a}$ & Paturel et al. (1997) & $\mathrm{IR}$ \\
$13 \mathrm{~b}$ & Paturel et al. (1997) & $\mathrm{HI}$ \\
$14 \mathrm{a}$ & NASA/IPAC Extragalactic Database (NED) IR \\
$14 \mathrm{~b}$ & NASA/IPAC Extragalactic Database (NED) HI \\
$14 \mathrm{c}$ & NASA/IPAC Extragalactic Database (NED) X \\
15 & Nishiyama \& Nakai (2001) & $\mathrm{CO}$ \\
16 & O'Sullivan et al. (2001) & $\mathrm{X}$ \\
$17 \mathrm{a}$ & Roberts et al. (1991) & $\mathrm{IR}$ \\
$17 \mathrm{~b}$ & Roberts et al. (1991) & $\mathrm{HI}$ \\
$17 \mathrm{c}$ & Roberts et al. (1991) & $\mathrm{CO}$ \\
$17 \mathrm{~d}$ & Roberts et al. (1991) & $\mathrm{X}$ \\
$18 \mathrm{a}$ & Sage 1993 & $\mathrm{HI}$ \\
$18 \mathrm{~b}$ & Sage 1993 & $\mathrm{CO}$ \\
19 & van Driel et al. (2000) & $\mathrm{HI}$ \\
$20 \mathrm{a}$ & Welch \& Sage (2003) \\
$20 \mathrm{~b}$ & Welch \& Sage (2003) & $\mathrm{HI}$ \\
21 & Wiklind et al. ((1995) \\
22 & Young et al. (1995) & $\mathrm{CO}$ \\
\hline & & $\mathrm{CO}$ \\
\hline & & $\mathrm{CO}$ \\
\hline & & \\
16 & &
\end{tabular}




\section{References}

Andreani, P., Casoli, F., \& Gerin, M. 1995, A\&A, 300, 43

Arp, H. 1966, Pasadena: California Inst. Technology

Arp, H. C., \& Madore, B. F. 1987, A Catalogue of Southern peculiar galaxies and associations, vol. 1 (Cambridge Univ. Press), 1

Bertola, F., \& Galletta, G. 1978, ApJ, 226, L115

Bettoni, D., Galletta, G., García-Burillo, S., \& Rodríguez-Franco, A. 2001, A\&A, 374, 421 (Paper I)

Beuing, J., Döbereiner, S., Böhringer, H., \& Bender, R. 1999, MNRAS, 302, 209

Boselli, A., Gavazzi, G., Lequeux, J., et al. 1995, A\&A, 300, L13

Bregman, J. N., Hogg, D. E., \& Roberts, M. S. 1992, ApJ, 387, 484

Burstein, D., Jones, C., Forman, W., Marston, A. P., \& Marzke, R. O. 1997, ApJS, 111, 163

Casoli, F., Boisse, P., Combes, F., \& Dupraz, C. 1991, A\&A, 249, 359

Casoli, F., Sauty, S., Gerin, M., et al. 1998, A\&A, 331, 451

de Vaucouleurs, G., de Vaucouleurs, A., Corwin H. G., et al. 1991, Third Reference Catalogue of Bright Galaxies (RC3) (New York: Springer-Verlag)

Fabbiano, G., Kim, D.-W., \& Trinchieri, G. 1992, ApJS, 80, 531

Feigelson, E. D., \& Nelson, P. I. 1985, ApJ, 293, 192

Galletta, G. 1996, in Barred Galaxies, IAU Coll 117, ed. R. Buta, D. A. Crocker, \& B. G. Elmegreen, ASP Conf. Ser., 91, 429

Gerin, M., \& Casoli, F. 1994, A\&A, 290, 49

Hawarden, T. G., Longmore, A. J., Tritton, S. B., Elson, R. A. W., \& Corwin, H. G., Jr. 1981, MNRAS, 196, 747

Horellou, C., Casoli, F., \& Dupraz, C. 1995, A\&A, 303, 361
Knapp, G. R., Guhathakurta, P., Kim, D.-W., \& Jura, M. 1989, ApJS, 70,329

Lavezzi, T. E., Dickey, J. M., Casoli, F., \& Kazès, I., 1999, AJ, 117, 1995

Nishiyama, K., \& Nakai, N. 2001, PASJ, 53, 713

Ochsenbein, F., Bauer, P., \& Marcout, J. 2000, A\&AS, 143, 23

O’Sullivan, E., Forbes, D. A., \& Ponman, T. J. 2001, MNRAS, 328 461

Paturel, G., Andernach, H., Bottinelli, L., et al. 1997, A\&AS, 124, 109

Roberts, M., Hogg, D. E., Bregman, J. N., Forman, W. R., \& Jones, C. 1991, ApJS, 75, 751

Sage, L. J. 1993, A\&A, 100, 537

Sage, L. J. 1993, A\&A, 272, 123

Schweizer, F., Whitmore, B. C., \& Rubin, V. C. 1983, AJ, 88, 909

Strong, A. W., Bloemen, J. B. G. M., Dame, T. M., et al. 1988, A\&A, 207,1

van Driel, W., Ragaigne, D., Boselli, A., Donas, J., \& Gavazzi, G. 2000, A\&AS, 144, 463

Veron-Cetty, M. P., \& Veron, P. 2000, ESO Scient. Rep. 19, 1

Vorontsov-Velyaminov, B. A. 1959, Atlas and catalog of interacting galaxies

Welch, G. A., \& Sage, L. J. 2003, ApJ, 584, 260

Whitmore, B. C., Lucas, R. A., McElroy, D. B., et al. 1990, AJ, 100, 1489

Wiklind, T., Combes, F., \& Henkel, C. 1995, A\&A, 297, 643

Young, J. S., Xie Shuding, Tacconi, L. J., et al. 1995, ApJS, 98, 219 